\title{
The Decision Decoding ToolBOX (DDTBOX) - A Multivariate Pattern Analysis Toolbox for Event-Related Potentials
}

\author{
Stefan Bode ${ }^{1} \cdot$ Daniel Feuerriegel ${ }^{1,2}$ (1) $\cdot$ Daniel Bennett ${ }^{1,3} \cdot$ Phillip M. Alday ${ }^{2,4}$ \\ Published online: 2 May 2018 \\ (C) The Author(s) 2018
}

\begin{abstract}
In recent years, neuroimaging research in cognitive neuroscience has increasingly used multivariate pattern analysis (MVPA) to investigate higher cognitive functions. Here we present DDTBOX, an open-source MVPA toolbox for electroencephalography (EEG) data. DDTBOX runs under MATLAB and is well integrated with the EEGLAB/ERPLAB and Fieldtrip toolboxes (Delorme and Makeig 2004; Lopez-Calderon and Luck 2014; Oostenveld et al. 2011). It trains support vector machines (SVMs) on patterns of event-related potential (ERP) amplitude data, following or preceding an event of interest, for classification or regression of experimental variables. These amplitude patterns can be extracted across space/electrodes (spatial decoding), time (temporal decoding), or both (spatiotemporal decoding). DDTBOX can also extract SVM feature weights, generate empirical chance distributions based on shuffled-labels decoding for group-level statistical testing, provide estimates of the prevalence of decodable information in the population, and perform a variety of corrections for multiple comparisons. It also includes plotting functions for single subject and group results. DDTBOX complements conventional analyses of ERP components, as subtle multivariate patterns can be detected that would be overlooked in standard analyses. It further allows for a more explorative search for information when no ERP component is known to be specifically linked to a cognitive process of interest. In summary, DDTBOX is an easy-to-use and open-source toolbox that allows for characterising the time-course of information related to various perceptual and cognitive processes. It can be applied to data from a large number of experimental paradigms and could therefore be a valuable tool for the neuroimaging community.
\end{abstract}

Keywords Multivariate pattern classification analysis (MVPA) - Decoding - Event-related potentials (ERPs) . Electroencephalography $(\mathrm{EEG}) \cdot$ Toolbox $\cdot$ Support vector machines

\section{General Introduction}

In recent years, the use of multivariate pattern analysis (MVPA) techniques for neuroimaging data has rapidly increased.

Stefan Bode and Daniel Feuerriegel contributed equally to this publication and are shared first authors.

Daniel Feuerriegel

dfeuerriegel@unimelb.edu.au

1 Melbourne School of Psychological Sciences, The University of Melbourne, Melbourne, Australia

2 School of Psychology, Social Work and Social Policy, University of South Australia, Adelaide, Australia

3 Princeton Neuroscience Institute, Princeton University, Princeton, New Jersey, USA

4 Max Planck Institute for Psycholinguistics, Nijmegen, The Netherlands
Beginning with Edelman and colleagues' (1998) and Haxby and colleagues' (2001) seminal studies, applications of MVPA to functional magnetic resonance imaging (fMRI) data have become increasingly popular, leading to a strong trend towards investigating representations and predicting "information" (i.e. the content of cognition) in cognitive neuroscience (for reviews and comments see: Davis and Poldrack 2013; Haynes 2015; Haynes and Rees 2006; Heinzle et al. 2012; Hogendoorn 2015; Mur et al. 2009; Norman et al. 2006; Kriegeskorte et al. 2006; Tong and Pratte 2012; Woolgar et al. 2014, 2016) and to the publication of several toolboxes (Hanke et al. 2009a; Hebart et al. 2015; Oosterhof et al. 2016). Most variants of MVPA have in common that (usually supervised) classifiers are trained to predict the content of cognitive processes directly from local spatial activation patterns, as measured by the blood-oxygen-level-dependent (BOLD) signal. Due to the poor temporal resolution of fMRI, however, it is often difficult to track any fast decision processes in time, and to determine the timecourse with which predictive information regarding these processes is represented in neural data. 
One potential solution to this problem is to apply MVPA to electroencephalography (EEG) data, as EEG has a far better temporal resolution in the range of milliseconds, as opposed to seconds in fMRI (other techniques such as magnetoencephalography, MEG, will not be discussed here; for reviews see King and Dehaene 2014; Grootswagers et al. 2016). Multivariate analysis techniques were already applied to EEG data to investigate cognition several decades ago (Gevins et al. 1979), but are currently experiencing a strong revival (for reviews and technical comments see: Bai et al. 2007; Blankertz et al. 2011; Contini et al. in press; King and Dehaene 2014; Parra et al. 2005; Sajda et al. 2009; Stokes et al. 2015). By taking advantage of the multivariate nature of EEG signals, multivariate analysis techniques can, for example, predict the outcomes of decisions, parameters of decision models, and decision errors directly from activity patterns (e.g., Blank et al. 2013; Bode et al. 2012, 2014; Bode and Stahl 2014; Boldt and Yeung 2015; Charles et al. 2014; Chung et al. 2015; Das et al. 2010; Parra et al. 2002; Philiastides and Sajda 2006; Philiastides et al. 2006; Ratcliff et al. 2009; Tzovara et al. 2015; Van Vugt et al. 2012; for related approaches see: El El Zein et al. 2015; Wyart et al. 2012, 2015). Others have used similar techniques to investigate visual awareness (Hogendoorn et al. 2015; Hogendoorn and Verstraten 2013; Fahrenfort et al. 2017), multisensory integration (Chan et al. 2017), and automatic processing of semantic features of task-irrelevant stimuli (Bode et al. 2014). MVPA has also been extensively used for constructing brain-computer interfaces (BCIs; e.g., Müller et al. 2004, 2008).

We present a novel open-source toolbox for MATLAB the Decision Decoding ToolBOX (DDTBOX) - that performs MVPA on the high-temporal-resolution EEG data typically analysed using univariate analyses of event-related potentials (ERPs). However, instead of analysing signals at single electrodes (i.e. channels), or averaging across a group of selected electrodes, for which ERP components have been described and linked to specific cognitive processes (e.g., Luck 2005), MVPA is applied to data from all electrodes in a predefined analysis time window, which thereby serves as multivariate input to a classifier (Parra et al. 2005). Such patterns of amplitude data can be extracted across space (e.g., from data averaged over a time window for each channel: spatial decoding), time (e.g., from all timepoints for each channel separately: temporal decoding), or both (from all timepoints for all channels: spatiotemporal decoding). This approach is arguably often more data- rather than hypothesis-driven compared to conventional ERP analyses, and has several benefits: First, even subtle multivariate EEG patterns that differ between experimental conditions can be detected that would otherwise be overlooked (Bode et al. 2012). Second, singletrial patterns of activity can be directly linked to parameters in decision models (e.g., Philiastides and Sajda 2006; Ratcliff et al. 2009; Tzovara et al. 2015), or used to predict subjective properties of stimuli such as arousal (e.g., Bode et al. 2014). Finally, MVPA on ERPs allows for a more explorative search for information when no ERP component is known to be specifically linked to the cognitive process of interest; it does not require a priori knowledge of the location and timing of an effect, which can vary substantially across experiments (discussed in Groppe et al. 2011).

We will now introduce the functionality of DDTBOX, which can be applied to data from a variety of experimental paradigms (and is by no means restricted to decision-making research). DDTBOX requires minimal experience with MATLAB coding, and integrates well with EEGLAB/ ERPLAB (Delorme and Makeig 2004; Lopez-Calderon and Luck 2014) and FieldTrip (Oostenveld et al. 2011), allowing users to prepare data using standard pre-processing pipelines for ERP analyses with only minimal additions. DDTBOX can, however, also use data preprocessed with many other commercially available software packages. In the following, we will first briefly discuss basic principles of classification approaches for the study of EEG signals. Then, we will provide an overview of the general architecture of the DDTBOX, complementing our detailed online user manual (https:// github.com/DDTBOX/DDTBOX/wiki). This will be followed by a user-oriented introduction to using DDTBOX, covering the general principles and features, the functional structure of the toolbox, and a section on how to prepare EEG data and how to configure analyses in DDTBOX. We will then provide examples of research which has already used beta-versions of DDTBOX, as well as limitations of analyses offered by DDTBOX. Finally, we will conclude by giving an outlook into planned future developments and extensions, as well as options for users to directly contribute to the toolbox. Where relevant, we will make reference to the detailed, more technical documentation available online, which is designed as an additional hands-on guide to the DDTBOX. We have also provided an example dataset online that can be downloaded to learn how to use DDTBOX, available at https://osf.io/bndjg/. This dataset is under a GNU General Public License (GPL) 3.0, which guarantees user rights to share and modify the data.

\section{Classification Based on ERP Data}

Machine learning has recently gained strong popularity in systems neuroscience. In particular, a supervised-learning approach using support vector machines (SVMs) has proved to be a powerful tool for neuroimaging analysis (e.g., Haynes 2015; Grootswagers et al. 2016). The power of this approach is derived from the fact that in most experiments, experimenters know the categories of interest a priori. These categories of interest typically correspond to different experimental conditions or participant response types (e.g., participants 
make decisions regarding object categories, make errors versus correct responses, report different subjective experiences, etc.). The aim for analysis is then to find patterns of neural activity that distinguish these categories. For multivariate EEG signals, this would correspond to finding patterns in the signal across time and space (electrodes) that distinguish the categories of interest. While this approach is still correlative, in the sense that it seeks to identify patterns of covariance between neural data and latent cognitive variables, its great advantage is that the structure of the neural data need not map straightforwardly to known aspects of the cognitive variables. Instead, it is sufficient that the EEG signal patterns predict the cognitive variables, thereby permitting researchers to conclude that information regarding cognitive variables is present in the neural data, either decodable from specific electrodes, or from specific processing time windows (Yarkoni et al. 2017).

A detailed description of SVMs has been provided elsewhere (Cortes and Vapnik 1995; Burges 1998; Hastie et al. 2001). Put simply, the general principle of SVM classification is to construct a hyperplane (i.e. a decision boundary) in multidimensional feature space to optimally separate exemplars into different categories (i.e. neural data mapping onto different experimental conditions). The nearest exemplars to this hyperplane from each category are known as the support vectors. The further away these exemplars are from the separating hyperplane, the better the classification. To avoid circularity (Kriegeskorte et al. 2009), estimation of the hyperplane must be performed on data independent from test data (left-out data from the same experiment, or new data from an identical experiment), which is subsequently used to evaluate the model by assigning category "labels" to each exemplar in the test data set. This usually involves k-fold cross-validation, in which the data are divided into $k$ subsets. The classifier is trained on k-1 subsets and tested using the left-out subset. This procedure is then independently repeated with each subset serving as the test data set once while training on the others (for an example see Meyers and Kreiman 2011). The average accuracy across all cross-validation steps, often referred to as "classification accuracy" or "decoding accuracy", can then be treated as an index for whether information about the categories of interest was represented in this specific pattern of brain activity. Statistically, this question can be assessed by submitting classification accuracy values to statistical testing, either against a theoretical chance performance level (e.g., with two balanced classes, the expected chance level $=50 \%$ ), or against an empirical test distribution, e.g., by comparing against results of analyses using randomly-shuffled condition labels (e.g., Stelzer et al. 2013). Although SVM classification is binary in nature, it can easily be extended to more complex multi-class classification problems by combining results from all pair-wise class combinations or performing one-vs.-other comparisons.
DDTBOX can also perform a type of generalisation analysis, testing whether patterns of information that discriminate between categories are stable across experimental contexts. For this, the classifiers are trained on data from one context (e.g., correct and error responses in task A) and used to predict the classes from data from another context (e.g., correct and error responses in task B). Such an approach is known as a cross-condition classification analysis. This can reveal whether the neural patterns that discriminate between outcomes are consistent across different task or stimulus presentation conditions, as the classifier should only be able to generalise from one to the other if patterns are highly similar (for an example from fMRI, see Bode et al. 2013).

For cognitive variables of interest that are continuous rather than categorical (such as response times), an alternative to SVM classification is support vector regression (SVR). SVR allows for trial-by-trial values of a continuous variable to be mapped to predicted values of that variable. DDTBOX offers both options, as we will outline below.

\section{General Principles of DDTBOX}

In order to perform a classification analysis, DDTBOX first requires the user to define discrimination groups, corresponding to categories of interest. These could be experimental conditions (e.g., different object categories) but also participants' behaviour (e.g., correct and incorrect responses). The eventlocked ERP data, which is used for the analysis, has undergone all pre-processing steps such as artefact correction and is epoched into time periods of interest as for conventional ERP analyses. Events can be either exogenous, such as stimulus presentations, or endogenous, such as behavioural responses. The epochs of ERP data are then sorted with respect to the categories of interest, and each epoch is assigned a class label corresponding to its category.

\section{SVM Back-End Software, Types of Analysis, and Kernels}

DDTBOX's main function is to prepare exemplars of patterns of ERP data for each participant for SVM classification or regression analyses. To perform such analyses, DDTBOX interacts with existing machine learning software packages to perform classification/regression (similar to The Decoding Toolbox for fMRI; Hebart et al. 2015). The user can choose between the LIBSVM package (Chang and Lin 2011), which has been used by other toolboxes in the field (e.g., Hebart et al. 2015), and LIBLINEAR (Fan et al. 2008), a less flexible but faster implementation of commonly-used SVM classification and regression algorithms (see the online documentation for details). Several SVM analysis options are available, including different SVM fitting methods and kernels. We refer to the 
websites of these software packages for detailed explanations of these options (LIBSVM: https://www.csie.ntu.edu.tw/ ح cjlin/libsvm; LIBLINEAR: https://www.csie.ntu.edu.tw/ حcjlin/liblinear). For most research questions requiring classification, C-SVM (as implemented in LIBSVM) with a linear kernel and a default regularising parameter $\mathrm{C}=1$ appears to be adequate and standard in the field, and is therefore the default option in DDTBOX. For multivariate regression, DDTBOX uses SVR in LIBSVM with a linear kernel and regularisation parameter $\mathrm{C}=0.1$ as the default option.

\section{Analysis Time Window Width}

DDTBOX performs analyses using a moving window approach: the signals of interest during a prespecified analysis time window are extracted and analysed, and the analysis time window is then moved by a specified step-size through the epoch (depicted in Fig. 1A). The user can specify the analysis window width and step-size. The optimal analysis time window width depends on the research question of interest, as information relating to some cognitive processes might be better captured by longer analysis time windows, while other short duration cognitive processes might be better captured using short analysis time windows. Our own previous work has successfully utilised analysis time windows ranging from $10 \mathrm{~ms}$ (e.g., Bode and Stahl 2014) to as long as $80 \mathrm{~ms}$ (e.g., Bode et al. 2012).

\section{Analysis Time Window Step Size}

The analysis time window is moved through the trial at a userdefined step size, independently repeating decoding analyses each time with data from the new time window (depicted in Fig. 1A). The step size could be the same as the analysis window width to achieve non-overlapping analysis time windows (e.g., $10 \mathrm{~ms}$ windows moved in steps of $10 \mathrm{~ms}$ ). Alternatively, the step size could be finer than the window width (e.g., $20 \mathrm{~ms}$ windows moved in steps of $10 \mathrm{~ms}$ ), leading to partial overlap of analysis time windows. This can be useful, for example, when one is interested in relatively fast cognitive processes, which might occur with a finer temporal resolution than the window size and therefore be captured only partly by two consecutive larger analysis time windows.

\section{Spatial and/or Temporal Analyses}

DDTBOX users can elect to run spatial analyses (Fig. 1B), which involve averaging across all data points included in the chosen analysis time window for each channel. This procedure results in one data point per channel (number of channels $\times 1$ activity pattern). Alternatively, the user can choose to disregard spatial patterns and perform temporal analyses (Fig. 1C) using data from single channels. In this case, all other channels are ignored, and the data points for the selected channel that are included in the analysis time window (number of data points $\times 1$ ) are treated as the activity pattern of interest. This analysis does not investigate spatially distributed information, but instead focuses on information distributed in time for a given channel. This approach is complementary to spatial classification, but it does not make use of all available (spatial) information. Finally, one can consider both spatial information (over channels) and temporal information (over timepoints) within a chosen analysis time window as the activity pattern (spatiotemporal analyses; number of data points $x$ number of channels), as shown in Fig. 1D.

\section{Averaging}

DDTBOX further provides the user with the option to average across separate sets of exemplars first before training the classifier. The standard option is not to average, which means that usually each experimental trial (or a part of such) is treated as one exemplar for one of the classes of interest. This usually has the advantage of maintaining a large number of exemplars for training and testing. However, if data from a large number of trials are available, one might consider averaging across subsets of trials for the same reasons that averaging is performed to obtain grand average ERPs: to optimise the signal-to-noise ratio. For example, if the experiment was split into 10 separate blocks, one could use block-averaged data for each class instead of single trials (e.g., see Bode et al. 2012). This is similar to first obtaining beta-estimates, or 'regressors', for separate functional 'runs' in fMRI, and then performing MVPA on these estimates (representing the run-averaged model fit of a general linear model) instead of on single volumes from all trials. Averaging usually results in estimates of exemplars with a higher signal-to-noise ratio, and can improve classification performance in some cases (see Isik et al. 2014; Grootswagers et al. 2016).

\section{Feature Weight Analyses}

DDTBOX allows users to extract and analyse feature weights from the fitted SVM classifiers. Much as regression coefficients describe the contribution of each predictor to the dependent variable, feature weights in SVM describe the contribution of each feature in determining the decision boundary, i.e. separating classes. As such, feature weights are used in DDTBOX to estimate the relative importance of different features (e.g., channels in spatial decoding analyses) for classification or regression. Accordingly, feature weights are analysed in DDTBOX to identify sources of information that the classifier uses to distinguish between experimental categories of interest. The 'raw' feature weights derived from SVMs are prone to erroneous interpretations regarding the sources of information used for decoding, as they can be affected by 
Fig. 1 Decoding approaches in DDTBOX. (a) Example of the windowed analysis approach. DDTBOX performs MVPA on time windows of EEG data (time windows outlined in blue). For each analysis the time window is moved through the trial by a predefined step size. (b) Example of spatial decoding. For each channel EEG data is averaged across timepoints within the analysis time window, resulting in one value per channel used for MVPA. (c) Example of temporal decoding. MVPA is performed using data from each timepoint within the analysis time window, for each channel separately. (d) Example of spatiotemporal decoding. All timepoints at all channels are used in combination for MVPA
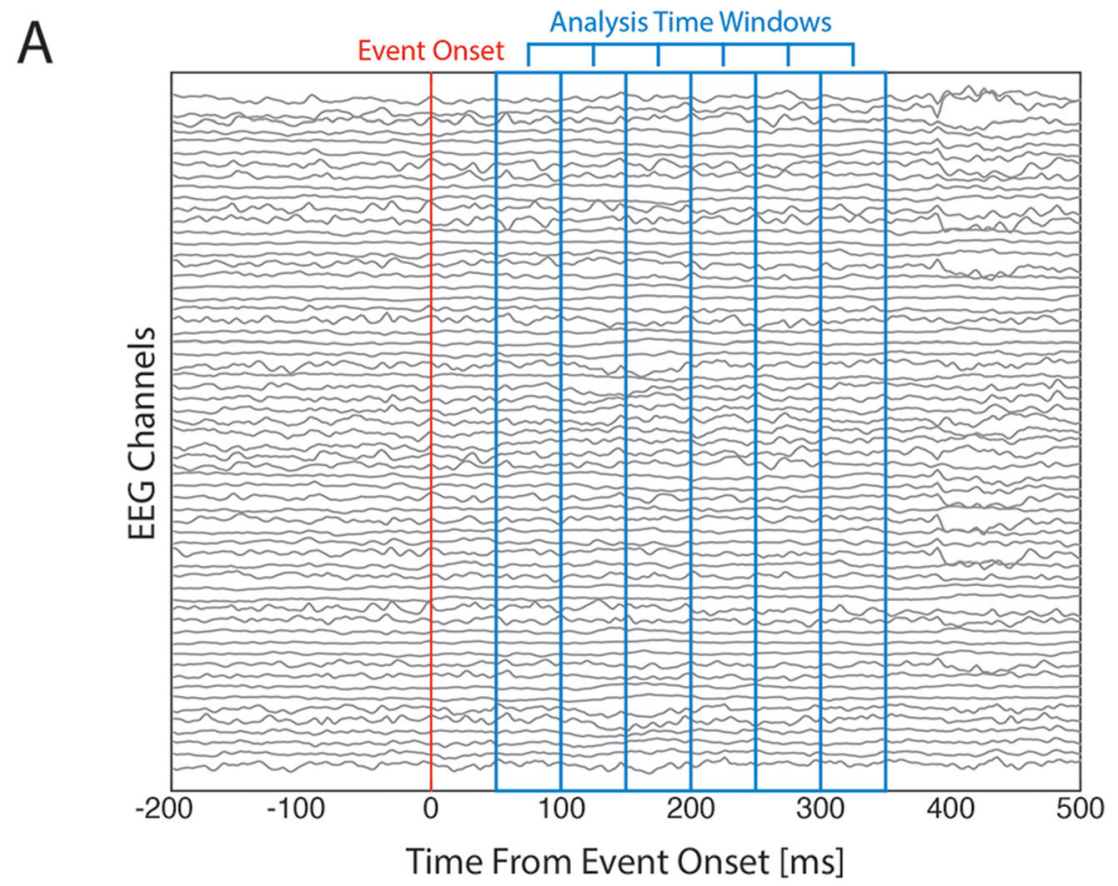

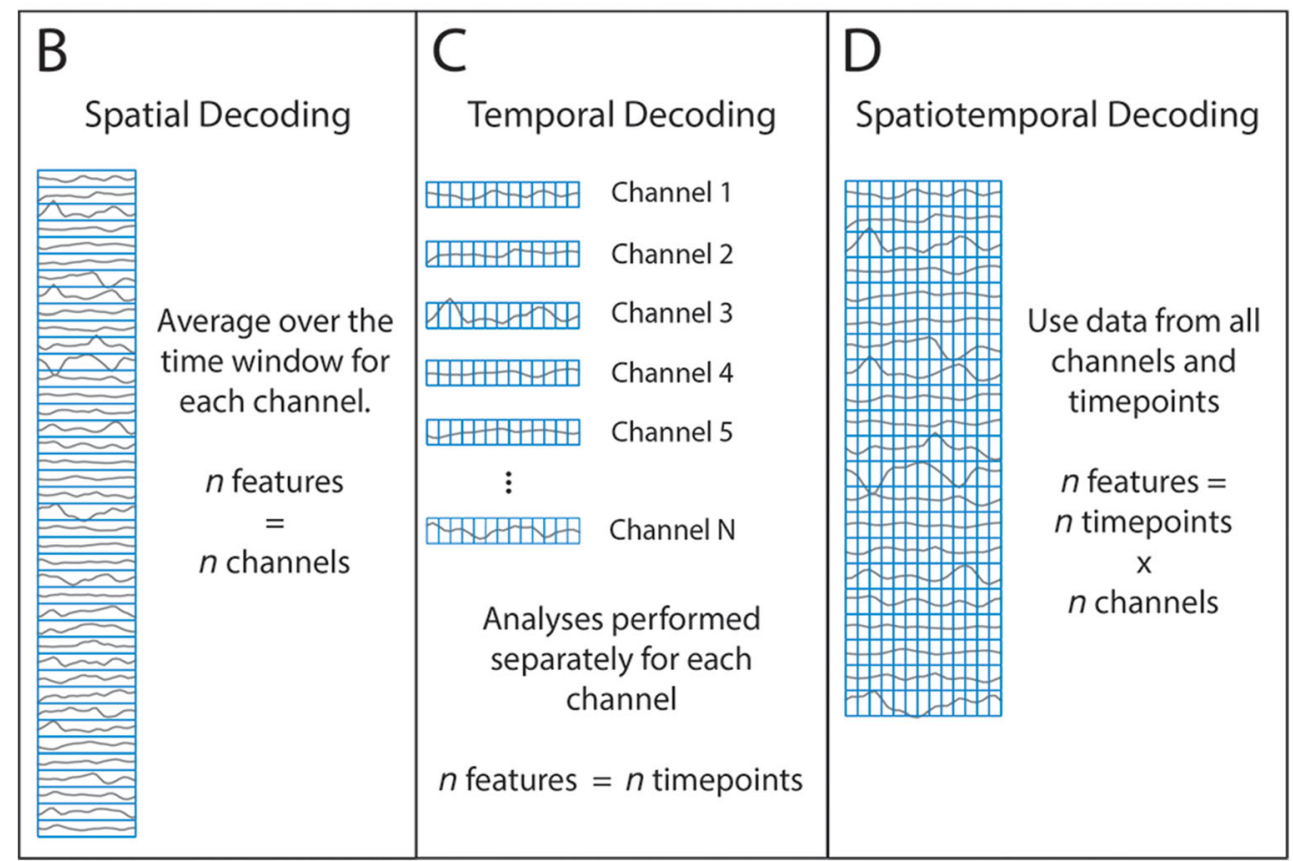

other statistically independent signals (such as noise generated by muscle activity, which as a feature may be strongly weighted but irrelevant). However, this can be corrected in DDTBOX by employing the algorithm described by Haufe et al. (2014).

In spatiotemporal analyses (see above) the features are single timepoints within the analysis time window for each channel. In DDTBOX feature weights are averaged across timepoints within each analysis window to output an averaged feature weight value for each channel (in consequence, group level feature weight analyses are only implemented for spatial and spatiotemporal analyses). Furthermore, as the sign of the feature weights indicate the importance of each feature for one or the other (arbitrary) category, and since the sign of each feature weight is therefore arbitrary, DDTBOX computes absolute feature weights, which indicate the importance for the classification in general (i.e. for either category). However, the advanced user can also access the original signed feature weights at individual timepoints within each analysis window. Lastly, feature weights from each analysis step are zstandardised to make them comparable between analyses. Hence, the final output is one absolute, z-standardised feature 
weight value for each channel for each analysis time window. These are used for group-level statistical testing (see below).

\section{Statistical Testing}

The result of each single analysis for each participant is a percentage value of correct classifications for all exemplars contained in the test-data set (for classification analyses), or a Fisher-Z transformed correlation between the predicted labels and the true labels (for regression analyses). Then, after the $\mathrm{k}$-fold cross-validation procedure, all $\mathrm{k}$ outcome values are averaged to index the overall accuracy. As it is theoretically possible that accuracy estimates were inflated by chance due to the random assignments of exemplars to training and test sets, the default option in DDTBOX is to re-compute the sets $\mathrm{m}$ times (i.e. a new, fully independent draw of $\mathrm{k}$ sets) and to repeat all analyses for a user-specified number of iterations. The default is to repeat all cross-validated analyses with independently drawn sets ten times. For example, choosing $\mathrm{k}=10$ for cross-validation, and $m=10$ iterations will result in $10 \times$ $10=100$ analyses, and the final accuracy will be the average of all 100 analyses. This procedure is designed to optimise reliability of results rather than accuracy values.

Statistical testing at a group level is then performed on average accuracy values obtained from the same analysis time window across participants. DDTBOX offers the option of testing against theoretical chance level (e.g., $50 \%$ for a balanced two-class classification, $33 \%$ for balanced 3-class classification, etc.). However, this approach has been criticised recently (Combrisson and Jerbi 2015). For example, increases in sample variance of accuracy values will also increase the chance of rejecting the null hypothesis when testing against theoretical chance (Allefeld et al. 2016). The default option in DDTBOX is therefore to estimate the empirical chance distribution by running decoding analyses on data with permuted condition labels. Specifically, DDTBOX repeats all original analyses (e.g., $\mathrm{m}$ iterations of a k-fold cross-validation procedure) with exactly the same data and the same category labels, but with assignment of labels to exemplars independently randomised for each iteration. This means that any potential biases in the original data (such as unbalanced numbers of exemplars across categories) also affect the permuted-label analyses. The original and the permuted-label analyses are otherwise completely identical, and the results of the permuted-label analyses can then be statistically compared to the original results.

Finally, group decoding accuracy at each analysis time window can be tested for statistical significance using either paired-samples $t$-tests or a group-level analysis method described in Allefeld et al. (2016) based on the minimum statistic (Friston et al. 1999). Importantly, both testing approaches do not provide population inference as do $t$-tests on univariate measures, but instead test the null hypothesis that there are at least some individuals within the sample that show above- chance decoding (i.e. is a fixed-effect analysis; discussed in Allefeld et al. 2016). However, the method based on the minimum statistic also provides lower bound estimates of the prevalence of decodable information in the population.

Analyses run in DDTBOX typically involve a large number of individual tests, requiring corrections for multiple comparisons to control the family-wise error rate. The number of tests performed depends on the number of analysis time windows, which can be minimised by selecting a restricted search space prior to running decoding analyses. DDTBOX offers a variety of correction techniques for multiple comparisons, some of which exploit temporal autocorrelation of the classification accuracy results across time windows to preserve statistical power. Available corrections include the Holm-Bonferroni method (Holm 1979), maximum statistic and cluster-based permutation tests (Blair and Karniski 1993; Maris and Oostenveld 2007), generalised family-wise error rate control (Korn et al. 2004) and false discovery rate control (e.g. Benjamini and Hochberg 1995; Benjamini et al. 2006). In addition, the distributional assumptions for paired-samples $t$-tests are often violated for samples of classification accuracy scores (Stelzer et al. 2013). DDTBOX can therefore also perform analyses using Yuen's paired-samples $t$-test (Yuen 1974; Wilcox 2012), which is more robust against violations of normality.

DDTBOX further offers group-level statistical testing of feature weights using paired-samples $t$-tests, with corrections for multiple comparisons over channels. Feature weights can be averaged over a number of analysis time windows before statistical testing, if required.

\section{Display Options}

DDTBOX allows plotting of the decoding performance and feature weight results at various stages. First, users can plot decoding accuracy scores (averaged over cross-validation steps and independent analyses) for individual subjects, for all analysis time windows (spatial and spatiotemporal analyses) or for all channels within a single time window (temporal analyses). For spatial and spatiotemporal analyses this is an 'information time-course', displaying the average accuracies (y-axis) for each chosen analysis time window ( $\mathrm{x}$ axis). Results of permuted condition labels analyses can also be plotted. This could be useful to quickly visually inspect the results for appropriateness of the chosen parameters (such as the window widths or step size), and also to confirm that the shuffled-label control analysis produces chance results. By contrast, temporal analysis results are plotted as a spatial map of accuracies for each channel, which are plotted as a heat map with a topographic projection onto the scalp.

Similarly, at a group level information time-course plots can be generated for spatial and spatiotemporal analyses, displaying the group-level accuracies (and optionally the permuted labels analysis results in the same plot) with error bars 
denoting standard errors of the mean. Users also have the option to include a vertical bar indicating the timing of the event of interest, as well as automatic marking of statistically significant analysis time windows based on a user-specified alpha level. Axis labels are automatically generated (based on the included baseline period and sampling rate, as well as minimum and maximum accuracy values) but can be manually modified, if desired. The temporal analyses group results are again heat maps displaying the colour-coded average group-level accuracy for each channel (note that standard errors are not included in this plot).

For the display of group-level feature weight maps (spatial and spatiotemporal analyses), two options are available. Firstly, a matrix of z-standardised, absolute feature weights per channel (y-axis) can be displayed for user-selected analysis time windows (x-axis). Secondly, the z-standardised, absolute group-level feature weights can be displayed for single analysis time windows or averages of user-specified analysis time windows. Feature weights can also be plotted as maps thresholded by statistical significance. All figures are plotted using MATLAB plotting routines, can be manually modified if desired, and exported to file formats including TIFF, JPG, PDF, EPS, and many others.

\section{Functional Structure of DDTBOX}

The functional structure of DDTBOX is extensively described in the wiki (https://github.com/DDTBOX/DDTBOX/wiki/ DDTBOX-Code-Structure) and will not be repeated here in detail. The order of data processing steps in DDTBOX for MVPA on single subject datasets is displayed in Fig. 2A. The operations performed in DDTBOX for group-level statistical testing are illustrated in Fig. 2B. Advanced users, who might want to gain access to data after specific processing steps, or who are considering expanding the toolbox at specific stages according to their needs, can use this information to easily navigate through the code.

The following section will only provide a brief overview of the functional structure, which is divided into phases:

Data Preparation (Phase 1). Includes preparation of the epoched data (see Section 5.1 below), as well as configuration of classification/regression analyses (as covered in the previous section).

Reading the data (Phase 2). This data is transformed into a MATLAB cell array with the following format:

eeg_sorted_cond\{run, cond\}(timepoints, channels, trials)

whereby run refers to the experimental block (if no separate blocks exist in the data, run will be 1), cond is the category/condition for classification (only one condition is used for support vector regression), timepoints are the single data points, channels the included EEG channels, and trials the single trials of the experiment. This is the general format for data storage, and each processing step will create a similar variable after the specified manipulations.

Reduction of data (Phase 3). Next, the data is reduced to the user-specified categories / conditions, which are used for the discrimination group of interest. This has the advantage that DDTBOX can operate within the memory constraints of most desktop computers.

Balancing the number of included trials (Phase 4). A frequent problem with classification analysis is that one might end up with an unequal number of trials per condition. This might be due to paradigms in which one condition is overrepresented (e.g., oddball paradigms, flanker tasks, or any other paradigm that requires more or less frequent events), responses of interest are not balanced (e.g., errors and correct responses, or most decision-making paradigms), or simply because by chance more trials are lost during EEG data preprocessing for one than for another condition. While this is not necessarily a problem for classification analyses, DDTBOX takes a conservative approach and equalises the number of trials per category / condition before classification.

Calculating block-average trials or pooling all trials across blocks (Phase 5). The next step involves averaging across trials (i.e. exemplars) within each experimental block, if this option was chosen. Alternatively, if there exist multiple blocks, but the user chose to treat them all as one long experiment, trials from each block are pooled at this stage.

Sorting for classification (Phase 6). The data is now sorted for the classification or regression process. For this, all trials (or block-averaged trials) will be divided into the userspecified number of $\mathrm{k}$ sets (the default is $\mathrm{k}=10$ ), which also specifies the number of cross-validation sets to be executed. For each full cross-validation cycle (repeated $\mathrm{m}$ times; the default is $\mathrm{m}=10$; see section 3.7 Statistical testing) trials are randomly assigned to one of the sets with the restriction that no set can have more trials than the others (left-over trials are excluded for this cycle). Of these sets, $\mathrm{k}-1$ are randomly assigned to the training data variable while the left-out set is assigned to the test data variable. All $\mathrm{k}$ combinations are stored before the random assignment of trials to sets and their sorting into training data and test data is performed again for all $\mathrm{m}$ iterations. For SVR, an additional matrix containing one value (the condition label) for each trial is used and substitutes for the class labels.

Vector preparation (Phase 7): After sorting data into training and test sets, DDTBOX extracts data from within the analysis time window and reshapes data from each trial into a single vector. These vectors are then used for training and testing the SVM classification or regression model. 


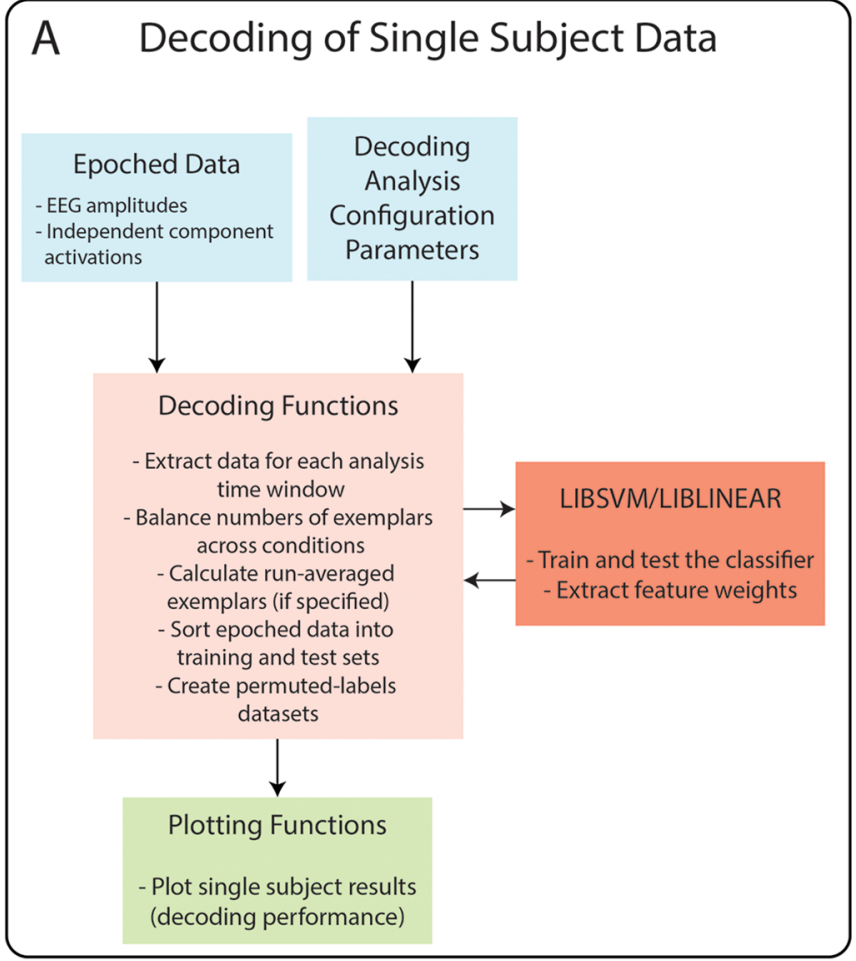

Fig. 2 Functional structure of DDTBOX. (a) The single subject data decoding functions accept epoched data and analysis configuration parameters. Epoched data is extracted for selected analysis time windows, and sorted for SVM classification or regression, for each cross-validation step and each independent analysis (full set of crossvalidation steps). SVM classification/regression is performed in LIBSVM or LIBLINEAR. (b) Group-level statistical analysis functions

\section{Using DDTBOX}

Preparing and running MVPA in DDTBOX involves four stages: preparing the data, configuring and running the decoding analyses, configuring and running group-level analyses, and plotting and interpreting the group results. Each of these are briefly described below.

\section{Preparation of EEG Data}

For decoding analyses DDTBOX uses epoched data, as described in section 4. Each participant dataset is saved as a separate data file. Epoched EEG data must be sorted by experimental condition and run/block, and then stored in this array. If applicable, SVR labels are stored within a separate cell array, with labels ordered in the same way as the corresponding epochs in the EEG data array. A function for automatically converting EEG data epoched using EEGLAB or ERPLAB is provided with the toolbox. This function can also extract epoched independent component activations in addition to EEG amplitudes. This function can further generate SVR labels files for each condition. Other data types (such as behavioural or steady-state visual evoked potential data)

\section{B Group-Level Statistical Analyses}

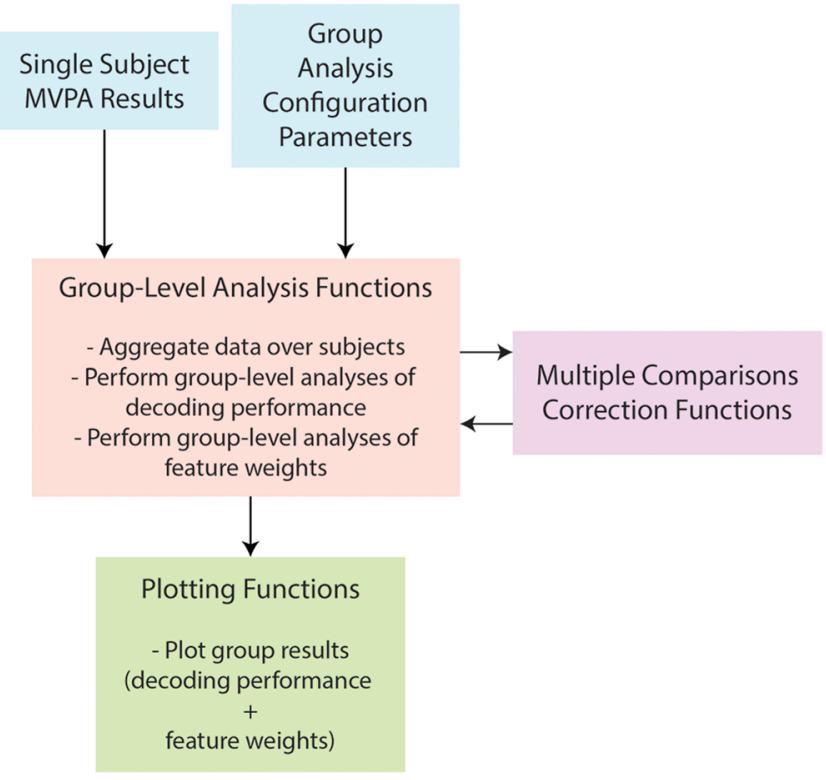

accept single subject MVPA results and group analysis configuration parameters. Decoding performance and feature weights are aggregated over single subjects and are statistically tested at the group level. Multiple comparisons corrections are applied as specified by the user. After analyses, DDTBOX can plot the group decoding accuracy and feature weights results

can, in principle, also be organised within the same cell array structure for use with DDTBOX by advanced users (for more information see the online documentation).

\section{Configuring and Running the Decoding Analyses}

DDTBOX uses a decoding analysis configuration script for defining all relevant parameters and running decoding analyses. Within this script the user can define single subject data filepaths, EEG dataset information, experimental conditions and discrimination groups, and a wide variety of multivariate classification/regression analysis parameters. Finally, the subjects and discrimination groups for analyses are defined, and the DDTBOX core decoding functions are called from this script. Users can copy and adapt these scripts for their own experiments; all parameters are clearly explained in the code comments of the script.

Once all the configuration parameters have been specified, the user can run decoding analyses by executing the MATLAB configuration script. SVM classification/ regression performance and feature weights information will be stored in a separate file for each subject. 


\section{Configuring and Running Group-Level Analyses}

Group-level statistical analyses of classification/regression performance and feature weights are configured and run using a group-level analysis configuration script. Within this script the user must define the filepaths of decoding results files, EEG dataset information, group-level statistical analysis and plotting parameters, and must specify the subjects and discrimination groups to use for analyses. Running this configuration script will perform all specified group-level statistical analyses on classification/regression performance and feature weights, which can also be plotted at this stage if desired.

\section{Plotting and Interpreting the Group Results}

DDTBOX offers a variety of plotting options for classification/ regression performance and feature weights results at the group level. These may be performed when running group-level statistical analyses, and can be replotted using a separate set of easy-to-configure plotting scripts.

For spatial and spatiotemporal decoding analyses group average classification/regression performance is plotted for each selected time window in the epoch, for results of both original and permuted labels decoding analyses (Fig. 3A). For temporal decoding analyses group average performance for a single analysis time window is plotted as a topographic heat map (Fig. 3B). Feature weights are also plotted in this way, and can also be plotted as a map thresholded for statistical significance (Fig. 3C).

\section{Toolbox Validation Using Simulated Data}

To demonstrate that the toolbox functions correctly we ran single subject decoding analyses using simulated EEG data. These analyses were designed to show that the basic functions of the toolbox work properly, rather than to evaluate all capabilities of the toolbox. We created a subject dataset consisting of 100 timepoints, 64 channels and 1000 epochs. Samples for each timepoint and channel in each epoch were sampled from independent Gaussian random noise (mean $=0$, standard deviation $=1$ ). A second dataset was created in the same way, except that a signal value of 0.05 was added to the first 10 channels for timepoints 51-100. Three more datasets were generated in this way, instead adding values of $0.1,0.2$ and 0.3 . All datasets contained Gaussian noise only at timepoints $1-50$, but differed (due to the addition of the signal values) at timepoints $51-100$. We note that temporally independent noise is not typical of real EEG data, but it sufficient for the purposes of our simulations.

We then performed spatial decoding using DDTBOX functions and $\mathrm{C}$-support vector classification as implemented in
LIBSVM to compare the first dataset to each of the other datasets with added signals. We used window and step sizes of $10 \mathrm{~ms}, 10$-fold cross-validation and 10 independent repetitions of cross-validated analyses. We also calculated absolute SVM feature weights corrected using the Haufe et al. (2014) method.

Fig. 4 displays the results of the validation analyses, showing chance-level classification performance during the first $50 \mathrm{~ms}$ of the simulated epoch where there are no systematic differences between the datasets. Classification accuracy then increases above chance from 51 to $100 \mathrm{~ms}$ according to the amount of signal (relative to the noise) in each dataset. Plots of feature weights for the time window 51-60 ms (when the signals were present) show that only the first 10 features (those containing the signal) have large weights, and that feature weights are larger for datasets with higher signal-to-noise ratios.

\section{Examples of Research Using DDTBOX}

In this section, we briefly review some studies that have used DDTBOX to investigate cognitive functions. We will use these to illustrate some recent research questions for which MVPA analysis has been profitably applied to ERP data; however, there are many other potential research questions for which DDTBOX could be used.

DDTBOX owes its name to its first application in perceptual decision-making (Bode et al. 2012). In this EEG study, images of pianos and chairs were presented after a $100 \mathrm{~ms}$ forward mask and longer backward mask (500 ms minus the duration of the target stimulus, which was either $16.7 \mathrm{~ms}, 33.3 \mathrm{~ms}, 50 \mathrm{~ms}$, or $66.7 \mathrm{~ms}$ ). A randomised response mapping screen was shown after the backward mask, circumventing early motor preparation. DDTBOX was used to predict the displayed object category, as well as participants' category choices, at all four discriminability levels. First, a spatial classification approach was applied, using $80 \mathrm{~ms}$ analysis time windows moved in steps of $20 \mathrm{~ms}$. It was found that the spatial patterns of EEG data predicted the displayed as well as the chosen category during the presentation of the poststimulus mask, with decreasing accuracy and fewer predictive time windows with decreasing discriminability of the objects (Bode et al. 2012). The study also presented phaserandomised visual noise images at the shortest presentation duration $(16.7 \mathrm{~ms})$, but participants believed themselves to be guessing real object categories. Participants' choices could be predicted from activity patterns from the pre-stimulus time period. This was interpreted as brain activity reflecting pre-existing decision biases resulting from carry-over effects of decisions in previous trials. To identify channels likely to contain this predictive information, complementary temporal classification analyses, using data from each channel separately, were performed for selected time windows showing high group classification accuracy in the spatial decoding analysis. Temporal decoding 

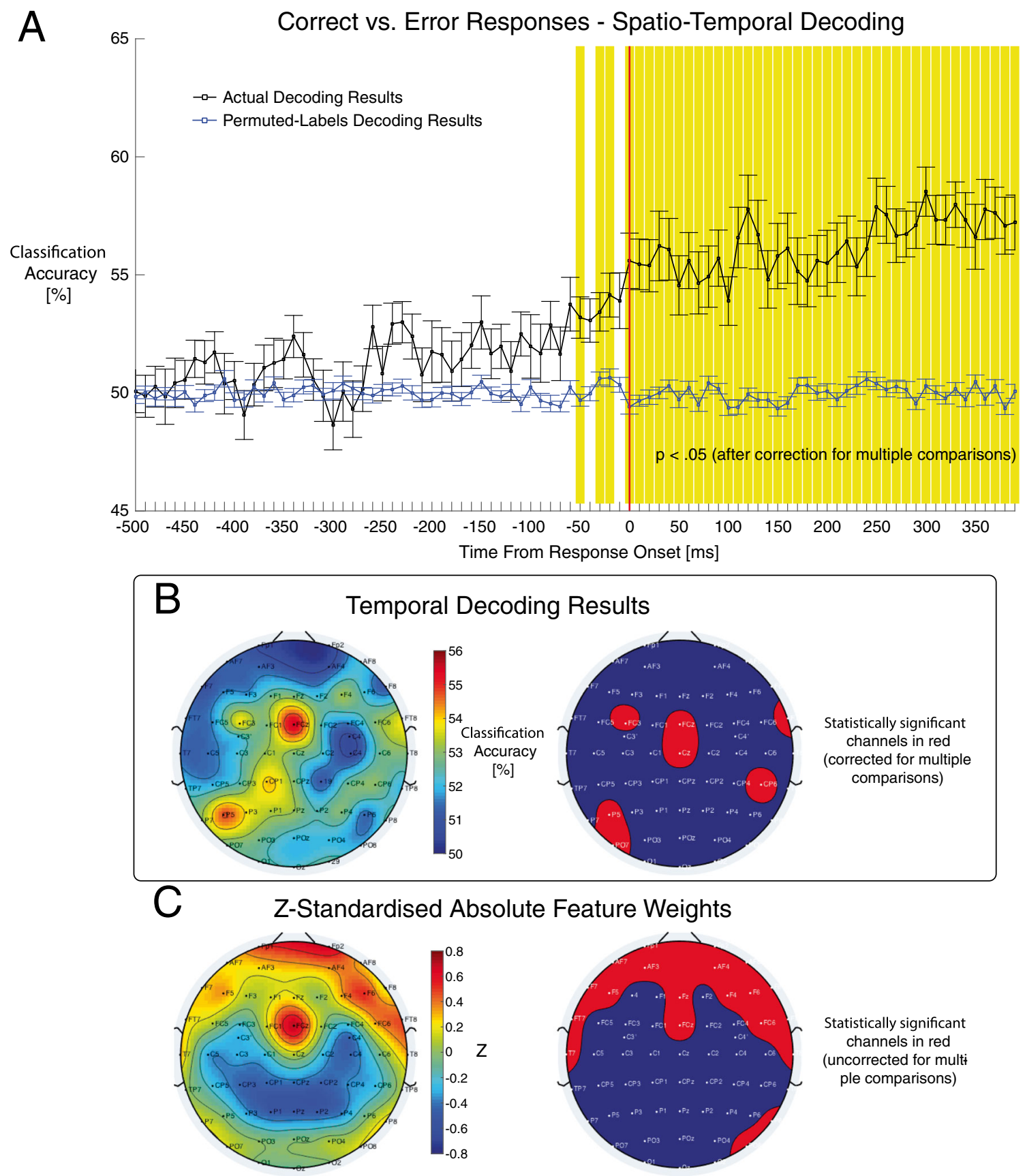

Fig. 3 Examples of group-level results outputs produced by DDTBOX. (a) Group average classification accuracy scores by time window from response onset. The black line represents the actual decoding results, blue line is the permuted-labels analysis results. Error bars represent standard errors of the mean. Shaded time windows are statistically significant after correction for multiple comparisons. (b) Temporal decoding results. A single time window was selected for temporal decoding analyses (100$300 \mathrm{~ms}$ from response onset). This time range approximates the timing of the error positivity ERP component in Bode and Stahl (2014). The left

analyses showed that channels predominantly over the visual cortex encoded object information early after stimulus presentation, while prefrontal channels did so during later stages before response preparation. For the pure-noise condition, decision- scalp map plots group average classification accuracy for each channel. The map on the right highlights in red the channels showing decoding accuracy scores that were statistically significantly above zero. (c) Feature weights results averaged over time windows spanning 100-300 ms from response onset. The left scalp map displays group averages of zstandardised absolute feature weights. The map on the right highlights in red the feature channels with feature weights with z-scores that were significantly above zero

related information was found for both channels over visual cortex and prefrontal cortex during the pre-stimulus period. Taken together, these results demonstrate that the classification analyses as implemented in DDTBOX can indeed detect subtle decision- 
A

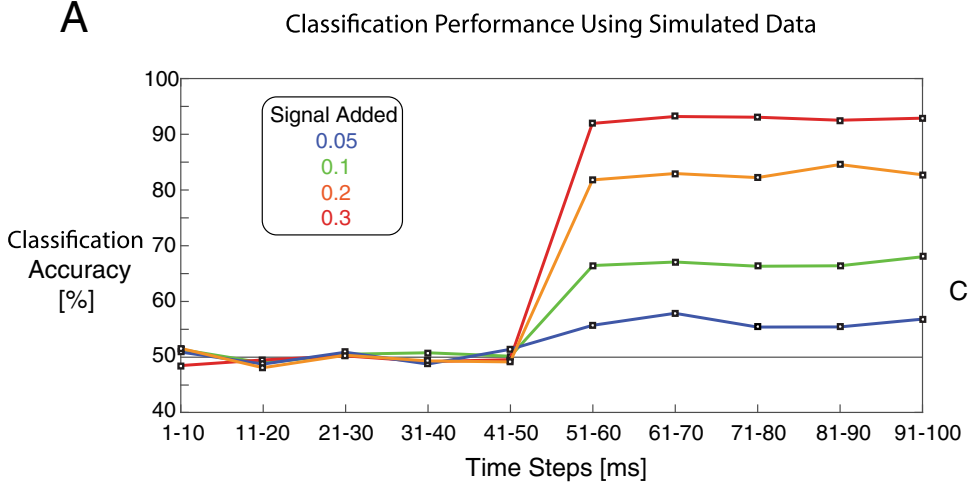

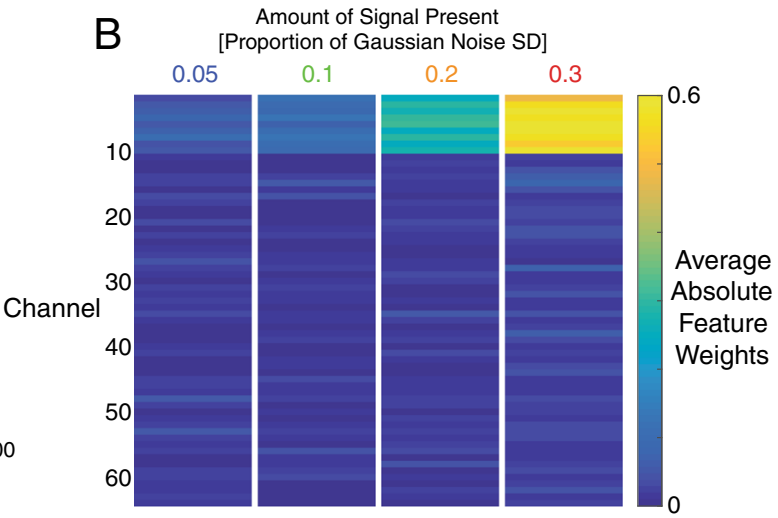

Amount of Signal Presen

[Proportion of Gaussian Noise SD]
Fig. 4 Results of toolbox validation analyses using simulated data. (a) Classification accuracies for four separate analyses, each classifying between one noise dataset (consisting of independent samples of Gaussian noise, mean $=0, \mathrm{SD}=1$ ) and one signal dataset consisting of Gaussian noise plus a signal. This signal consisted of values $0.05,0.1,0.2$ or 0.3 added to the first 10 channels during timepoints 51-100. Accuracy

related information, which would have gone unnoticed in conventional ERP analyses. In contrast to the MVPA results, no ERP components selected for analyses showed differential activity related to piano and chair decisions, or differences in prestimulus baseline activity by category decision for the pure noise condition. This is likely due to subject-specific patterns of EEG activity that differ in response to pianos and chairs, which may not be consistent across subjects and 'average-out' in conventional univariate ERP analyses.

In another EEG study, spatiotemporal classification was performed using DDTBOX to predict whether an upcoming response for a parity decision in a speeded digit flanker task was correct or erroneous (Bode and Stahl 2014). Participants were asked to indicate, using one of two response buttons, whether a central digit on the screen was odd or even, in the presence of two flanker digits on each side that were also odd or even, thereby creating congruent or incongruent decision conditions. For MVPA $10 \mathrm{~ms}$ analysis time windows were used, moved in steps of $10 \mathrm{~ms}$ through the trial, approaching the behavioural response onset. MVPA revealed that EEG activity patterns from $100 \mathrm{~ms}$ before response execution already predicted whether the upcoming response would be erroneous, while conventional ERP analyses found that the error-related negativity (ERN), which follows a response by $80-100 \mathrm{~ms}$, was the first ERP component to predict decision errors (Bode and Stahl 2014). Follow-up analyses of feature weights suggested that this early information originated from channels over visual and motor cortices. In this study classification analyses performed using DDTBOX provided information related to decision errors preceding the participants' responses, and informed theories of how information about upcoming decision errors could accumulate over time to support online error monitoring processes (Bode and Stahl 2014).

DDTBOX has also been used to investigate perceptual categorisation of faces (Quek and Rossion 2017) and multi-sensory integration in elderly and younger scores were averaged across 10 cross-validation steps and 10 analysis repetitions. (b) Absolute SVM feature weights during time window 51$60 \mathrm{~ms}$, averaged over cross-validation steps and analysis repetitions. Larger feature weights are visible for channels 1-10 in datasets with larger signals relative to the noise

adults (Chan et al. 2017). Another application of DDTBOX has been the use of SVR to predict postexperimental ratings of affective and abstract stimulus attributes of task-irrelevant images to inform theories of automatic processing of stimulus features during passive exposure (Bode et al. 2014; Turner et al. 2017). These latter examples demonstrate that DDTBOX is by no means restricted to applications in decision-making. On the contrary, it lends itself to many possible questions for which conventional ERP analyses might not be suited, such as cases in which the specific encoding patterns and the timing of these patterns are unknown prior to the experiment.

\section{Versioning and Release Management}

To denote releases we use semantic versioning with the version number format X.Y.Z (https://semver.org/spec/v2.0.0. $\mathrm{html}$ ). $\mathrm{X}$ denotes the major version number (e.g. v.1.0.0, v2. 0.0 ). Changes to $X$ indicate backwards-incompatible changes to the structure of the toolbox code. Changes to $\mathrm{Y}$ denote a minor version release (e.g. v1.1.0, v1.2.0) indicating added features or capabilities. Changes to $\mathrm{Z}$ denote the patch version releases (e.g. v.1.0.1, v1.0.2) which include bugfixes and code documentation modifications. The current toolbox release is $\mathrm{v}$. 1.0.3, which incorporates minor bugfixes following the initial public release.

The numbered releases are tracked via Zenodo (https://doi. org/10.5281/zenodo.593871), which archives a snapshot of the code and assigns each release a DOI, allowing for users to cite specific versions and guaranteeing the later availability of those versions. This serves to support reproducible analyses. 


\section{Comparisons to other Packages for Time-Series Multivariate Data Analysis}

Other toolboxes have also been developed for MVPA on EEG and neuroimaging data. Below we outline the most influential toolboxes in the field and offer broad comparisons to DDTBOX in terms of analysis options and compatibility with EEG datasets. Given that there is active development of DDTBOX and other toolboxes, any limitations of each toolbox may be overcome in the near future.

CoSMoMVPA (Oosterhof et al. 2016) is a toolbox for MVPA of fMRI and M/EEG data running in MATLAB and OCTAVE. CoSMoMVPA offers support for a wide variety of MVPA methods, including some classification-based analyses not yet available in DDTBOX, such as temporal generalisation and representational similarity analysis (Kriegeskorte et al. 2008). However, CoSMoMVPA does not offer multivariate regression methods such as SVR or analyses of feature weights. DDTBOX also offers more extensive functionality for visualising results and performing group-level analyses. CoSMoMVPA has extensive documentation and tutorial material, but requires more advanced programming skills compared with DDTBOX. MNE-Python (Gramfort et al. 2013) also provides support for single-subject MVPA analyses, with direct support for temporal generalization and spatial decoding via spatial filters (Common Spatial, Effect-Matched-Spatial Filter). MNE-Python has extensive documentation and tutorial material; however, it is nearly all focused on single-subject analyses with a strong MEG bias. While MNE-Python offers great flexibility it also requires advanced programming skills.

In addition, there are several MVPA toolboxes specialised for fMRI data with a more extensive range of analysis options than DDTBOX, including The Decoding Toolbox (Hebart et al. 2015), The Princeton MVPA Toolbox (http://code.google.com/ $\mathrm{p} /$ princeton-mvpa-toolbox/), Pattern Recognition of Neuroimaging Toolbox (PRoNTo; Schrouff et al. 2013) and the RSA Toolbox (Nili et al. 2014) written in MATLAB, and PyMVPA (Hanke et al. 2009b) written in Python. These could, in principle, be applied to EEG data by those with advanced programming skills. However, these toolboxes currently offer limited options for visualisation of time series MVPA results and preparation of EEG data for decoding analyses.

\section{Limitations, Future Developments and Extensions}

Although it includes a range of analysis options, the current version of DDTBOX is still limited in several ways. The first notable limitation is the support for MVPA using different types of input data. At this stage, DDTBOX can perform MVPA on frequency domain and time-frequency data, as well as component activations from principal components analysis (PCA) or independent components analysis (ICA). However, DDTBOX does not yet offer result plotting capabilities, or automatic conversion to DDTBOXcompatible data files, for these data types. Future support for these data types will widen the applicability of DDTBOX for use with different experimental designs, for example studies examining multivariate patterns of steadystate visual evoked potential (SSVEP) data (e.g., Jacques et al. 2016). In particular, decoding with principal or independent components may also help improve decoding accuracy compared to EEG amplitudes (Grootswagers et al. 2016).

Another current limitation of DDTBOX is its restriction to using the same analysis time window for training and testing. Others have suggested that one strength of the multivariate approach is that the temporal generalisability of patterns across time can be investigated (Meyers et al. 2008; Carlson et al. 2011; King and Dehaene 2014; Fahrenfort et al. 2017). For this, a classifier could be trained on data from one time window and then tested at other time windows to assess the duration for which the same training data successfully predicts the cognitive process (or content) of interest. By using all possible combinations of training and test data, a full generalisation matrix can be compiled that is informative about the temporal dynamics of cognition (c.f. Fig. 3 in King and Dehaene 2014; see also Hogendoorn 2015). Temporal generalisation analyses will be added to a future version of DDTBOX.

A final noteworthy limitation is that the user is required to extract epoched data from EEGLAB/ERPLAB, and to create a configuration script containing all necessary information about the study and planned analyses, before using DDTBOX. While we provide a userfriendly wiki, example configuration scripts, and functions for automatically extracting data epoched using EEGLAB/ERPLAB, the use of DDTBOX nevertheless requires some basic knowledge of MATLAB. Our aim is that the next release will also function as a plug-in for EEGLAB, providing users with a graphical user interface (GUI) within the EEGLAB environment to input all DDTBOX configuration parameters, and the option to use data directly from EEGLAB. However, we are confident that the current release will be of great benefit for the research community, and our toolbox can easily be handled without a GUI.

In addition to extensions planned by the core developers, user-contributed features are welcome and encouraged. DDTBOX users have actively contributed to 
the toolbox since the initial public release. Users have suggested new features, reported bugs and contributed code to extend toolbox capabilities. Procedures and guidelines for submitting community-contributed code are available at https:/github.com/DDTBOX/DDTBOX/ wiki/Contributing-to-DDTBOX.

To preserve the stability and usability of toolbox code we will also add a suite of unit tests to a future DDTBOX release. This test suite will input simulated data into each function and ensure that the code runs without errors, and that the function outputs match predetermined expected values. All user- and developer-made code modifications will need to pass these unit tests before they can be incorporated into the toolbox.

\section{User Support}

Technical support for DDTBOX users is available via our mailing list (https://www.freelists.org/list/ddtbox). Questions and discussion points can be posted to this list and will be answered by the core developers as well as the broader community of DDTBOX users. Bugs and requests for new features can also be reported via the issue tracker on Github (https://github.com/DDTBOX/DDTBOX/issues).

\section{Summary}

To conclude, DDTBOX is a freely available, opensource toolbox for MATLAB that can be used for multivariate pattern classification and regression analyses on spatial, temporal and spatiotemporal patterns of EEG data. It is useful for investigating cognitive processes related to decision-making, object categorisation, perception, and potentially many other cognitive phenomena. This class of predictive methods can be used in a more explorative and data-driven fashion than conventional ERP analyses. DDTBOX has been used in several published studies and allows for detecting even subtle information that might be overlooked by standard ERP analyses. DDTBOX incorporates a variety of statistical tests, and the option to perform permuted-labels analyses to generate empirical chance distributions. It also generates feature weight maps, which provide useful estimates of the origins of the decodable information. DDTBOX is released under a GNU General Public License (GPL) v.2.0, meaning that users are free to share, modify and extend the toolbox as desired.

DDTBOX and the respective documentation is available at: https://github.com/DDTBOX/DDTBOX.

The developers are working on improving DDTBOX on a regular basis. Users can subscribe to our mailing list and will be regularly updated about new releases and features. As the code is openly-available on GitHub, we invite all users to contribute to DDTBOX by submitting their own extensions and improvements. Authors of accepted contributions will be acknowledged in future releases. With DDTBOX, we are hoping to provide a useful toolbox for multivariate EEG analysis that can grow with the needs of researchers and new directions in the field, driven and developed further by an active community of users.

\section{Information Sharing Statement}

DDTBOX (RRID:SCR_015978) is freely-available at https:// github.com/DDTBOX/DDTBOX with the respective software documentation at https://github.com/DDTBOX/DDTBOX/wiki. Data used for generating Fig. 3 are available for download at https://osf.io/bndjg/. DDTBOX runs on MATLAB, available at http://www.mathworks.com/products/matlab.

Acknowledgements The DDTBOX was inspired by SB's work with Prof John-Dylan Haynes on MVPA for fMRI, and some features of the code were modelled from code developed in the Haynes lab. We acknowledge helpful input from Dr. Carsten Bogler and Dr. Chun Siong Soon during this time. We are further thankful for important conceptual input and improvements resulting from collaborative work with Prof Jutta Stahl, Dr. Simon Lilburn, Prof Philip L. Smith, Dr. Elaine Corbett, Dr. Carsten Murawski and Dr. Owen Churches.

Authors Contributions The DDTBOX has been developed and written by SB, with significant contributions by DB, DF and PMA. All authors contributed to the online documentation and developed the learning material. SB, DF wrote the paper. All authors contributed to and approved the final version of the paper and agreed to be accountable for the content of this work.

Funding SB was funded by an Australian Research Council Discovery Early Career Researcher Award (ARC DECRA DE140100350).

\section{Compliance with Ethical Standards}

Conflict of Interest The authors declare no conflict of interest. No payments were received by neither the institutions nor funding agencies to create this toolbox, and institutions and funding agencies had no input into the content of the work or the publication.

Open Access This article is distributed under the terms of the Creative Commons Attribution 4.0 International License (http:// creativecommons.org/licenses/by/4.0/), which permits unrestricted use, distribution, and reproduction in any medium, provided you give appropriate credit to the original author(s) and the source, provide a link to the Creative Commons license, and indicate if changes were made.

\section{References}

Allefeld, C., Görgen, K., \& Haynes, J. D. (2016). Valid population inference for information-based imaging: From the second-level t-test to prevalence inference. NeuroImage, 141, 378-392.

Bai, O., Lin, P., Vorbach, S., Li, J., Furlani, S., \& Hallett, M. (2007). Exploration of computational methods for classification of 
movement intention during human voluntary movement from single trial EEG. Clinical Neurophysiology, 118, 2637-2655.

Benjamini, Y., \& Hochberg, Y. (1995). Controlling the false discovery rate: A practical and powerful approach to multiple testing. Journal of the Royal Statistical Society Series B: Statistical Methodology, 57, 289-300.

Benjamini, Y., Krieger, A. M., \& Yekutieli, D. (2006). Adaptive linear step-up procedures that control the false discovery rate. Biometrika, 491-507.

Blair, R. C., \& Karniski, W. (1993). An alternative method for significance testing of waveform difference potentials. Psychophysiology, $30,518-524$

Blank, H., Biele, G., Heekeren, H. R., \& Philiastides, M. G. (2013). Temporal characteristics of the influence of punishment on perceptual decision making in the human brain. The Journal of Neuroscience, 33, 3939-3952.

Blankertz, B., Lemm, S., Treder, M., Haufe, S., \& Müller, K. R. (2011). Single-trial anal-ysis and classification of ERP components - A tutorial. NeuroImage, 56, 814-825.

Bode, S., \& Stahl, J. (2014). Predicting errors from patterns of eventrelated potentials preceding an overt response. Biological Psychology, 103, 357-369.

Bode, S., Sewell, D. K., Lilburn, S., Forte, J., Smith, P. L., \& Stahl, J. (2012). Predicting perceptual decision biases from early brain activity. The Journal of Neuroscience, 32, 12488-12498.

Bode, S., Bogler, C., \& Haynes, J. D. (2013). Similar neural mechanisms for guesses and free decisions. NeuroImage, 65, 456-465.

Bode, S., Bennett, D., Stahl, J., \& Murawski, C. (2014). Distributed patterns of event-related potentials predict subsequent ratings of abstract stimulus attributes. PLoS One, 9, e109070. https://doi.org/ 10.1371/journal.pone.0109070.

Boldt, A., \& Yeung, N. (2015). Shared neural markers of decision confidence and error detection. Journal of Neuroscience, 35, 3467-3484.

Burges, C. J. (1998). A tutorial on support vector machines for pattern recognition. Data Mining and Knowledge Discovery, 2, 121-167.

Carlson, T. A., Hogendoorn, H., Kanai, R., Mesik, J., \& Turret, J. (2011). High temporal resolution decoding of object position and category. Journal of Vision, 11. https://doi.org/10.1167/11.10.9.

Chan, Y. M., Pianta, M. J., Bode, S., \& McKendrick, A. M. (2017). Neural correlates of audiovisual synchrony judgements in older adults. Neurobiology of Aging, 55, 38-48.

Chang, C. C., \& Lin, C. J. (2011). LIBSVM: a library for support vector machines. ACM Transactions on Intelligent Systems and Technology, 2, 27.

Charles, L., King, J. R., \& Dehaene, S. (2014). Decoding the dynamics of action, intention, and error-detection for conscious and subliminal stimuli. The Journal of Neuroscience, 34, 1158-1170.

Chung, D., Yun, K., \& Jeong, J. (2015). Decoding covert motivations of free riding and cooperation from multi-feature pattern analysis of EEG signals. Social Cognitive and Affective Neuroscience, 10, $1210-1218$

Combrisson, E., \& Jerbi, K. (2015). Exceeding chance level by chance: The caveat of theoretical chance levels in brain signal classification and statistical assessment of decoding accuracy. Journal of Neuroscience Methods, 250, 126-136.

Contini, E. W., Wardle, S. G., \& Carlson, T. A. (in press). Decoding the time-course of object recognition in the human brain: From visual features to categorical decisions. Neuropsychologia.

Cortes, C., \& Vapnik, V. (1995). Support-vector networks. Machine Learning, 20, 273-297. https://doi.org/10.1007/BF00994018.

Das, K., Giesbrecht, B., \& Eckstein, M. P. (2010). Predicting variations of perceptual performance across individuals from neural activity using pattern classifiers. NeuroImage, 51, 1425-1437.

Davis, T., \& Poldrack, R. (2013). Measuring neural representations with fMRI: practices and pitfalls. Annals of the New York Academy of Sciences, 1296, 108-134.
Delorme, A., \& Makeig, S. (2004). EEGLAB: an open source toolbox for analysis of single-trial EEG dynamics including independent component analysis. Journal of Neuroscience Methods, 134, 9-21.

Edelman, S., Grill-Spector, K., Kushnir, T., \& Malach, R. (1998). Toward direct visualization of the internal shape representation space by fMRI. Psychobiology, 26, 309-321.

El Zein, M., Wyart, V., \& Grezes, J. (2015). Anxiety dissociates the adaptive functions of sensory and motor response enhancements to social threats. eLife, 4, e10274. https://doi.org/10.7554/eLife.10274.

Fahrenfort, J. J., van Leeuwen, J., Olivers, C. N., \& Hogendoorn, H. (2017). Perceptual integration without conscious access. Proceedings of the National Academy Sciences of the United States of America, 114, 3744-3749.

Fan, R. E., Chang, K.-W., Hsieh, C. J., Wang, X. R., \& Lin, C. J. (2008). LIBLINEAR: a library for large linear classification. Journal of Machine Learning Research, 9, 1871-1874.

Friston, K., Holmes, A., Price, C., Buchel, C., \& Worsley, K. (1999). Multisubject fMRI studies and conjunction analysis. NeuroImage, 10, 385-396.

Gevins, A. S., Zeitlin, G. M., Yingling, C. D., Doyle, J. C., Dedon, M. F., Schaffer, R. E., Roumasset, J. T., \& Yeager, C. L. (1979). EEG patterns during 'cognitive' tasks. I. Methodology and analysis of complex behaviors. Electroencephalography and Clinical Neurophysiology, 47, 793-703.

Gramfort, A., Luessi, M., Larson, E., Engemann, D., Strohmeier, D., Brodbeck, C., Goj, R., Jas, M., Brooks, T., Parkkonen, L., \& Hämäläinen, M. (2013). MEG and EEG data analysis with MNEPython. Frontiers in Neuroscience, 7. https://doi.org/10.3389/fnins. 2013.00267.

Grootswagers, T., Wardle, S. G., \& Carlson, T. A. (2016). Decoding dynamic brain patterns from evoked responses: A tutorial on multivariate pattern analysis applied to time series neuroimaging data. Journal of Cognitive Neuroscience, 29, 677-697.

Groppe, D. M., Urbach, T. P., \& Kutas, M. (2011). Mass univariate analysis of event-related brain potentials/fields I: A critical tutorial review. Psychophysiology, 48, 1711-1725.

Hanke, M., Halchenko, Y. O., Sederberg, P. B., Olivetti, E., Fründ, I., Rieger, J. W., Herrmann, C. S., Haxby, J. V., Hanson, S. J., \& Pollmann, S. (2009a). PyMVPA: A unifying approach to the analysis of neuroscientific data. Frontiers in Neuroinformatics, $3,3$. https://doi.org/10.3389/neuro.11.003.2009.

Hanke, M., Halchenko, Y. O., Sederberg, P. B., Hanson, S. J., Haxby, J. V., \& Pollman, S. (2009b). PyMVPA: A python toolbox for multivariate pattern analysis of fMRI data. Neuroinformatics, 7, 37-53.

Hastie, T., Tibshirani, R., \& Friedman, J. (2001). The elements of statistical learning. Heidelberg: Springer.

Haufe, S., Meinecke, F., Görgen, K., Dähne, S., Haynes, J. D., Blankertz, B., \& Bießmann, F. (2014). On the interpretation of weight vectors of linear models in multivariate neuroimaging. NeuroImage, 87, 96 110.

Haxby, J. V., Gobbini, M. I., Furey, M. L., Ishai, A., Schouten, J. L., \& Pietrini, P. (2001). Distributed and overlapping representations of faces and objects in ventral temporal cortex. Science, 293, 24252430.

Haynes, J. D. (2015). A Primer on Pattern-Based Approaches to fMRI: Principles, Pitfalls, and Perspectives. Neuron, 87, 257-270.

Haynes, J. D., \& Rees, G. (2006). Decoding mental states from brain activity in humans. Nature Review Neuroscience, 7, 523-534.

Hebart, M. N., Görgen, K., \& Haynes, J. D. (2015). The Decoding Toolbox (TDT): a versatile software package for multivariate analyses of functional imaging data. Frontiers in Neuroinformatics, 8 , 88. https://doi.org/10.3389/fninf.2014.00088.

Heinzle, J., Anders, S., Bode, S., Bogler, C., Chen, Y., Cichy, R. M., Hackmack, K., Kahnt, T., Kalberlah, C., Reverberi, C., Soon, S. C., Tusche, A., Weygandt, M., \& Haynes, J. D. (2012). 
Multivariate decoding of fMRI data - Towards a content-based cognitive neuroscience. e-Neuroforum, 3, 1-16.

Hogendoorn, H. (2015). From sensation to perception: Using multivariate classification of visual illusions to identify neural correlates of conscious awareness in space and time. Perception, 44, 71-78.

Hogendoorn, H., \& Verstraten, F. A. (2013). Decoding the motion aftereffect in human visual cortex. NeuroImage, 82, 426-432.

Hogendoorn, H., Verstraten, F. A., \& Cavanagh, P. (2015). Strikingly rapid neural basis of motion-induced position shifts revealed by high temporal-resolution EEG pattern classification. Vision Research, $113,1-10$.

Holm, S. (1979). A simple sequentially rejective multiple test procedure. Scandanavian Journal of Statistics, 6, 65-70.

Isik, L., Meyers, E. M., Leibo, J. Z., \& Poggio, T. (2014). The dynamics of invariant object recognition in the human visual system. Journal of Neurophysiology, 111, 91-102.

Jacques, C., Retter, T. L., \& Rossion, B. (2016). A single glance at natural face images generate larger and qualitatively different category-selective spatio-temporal signatures than other ecologically-relevant categories in the human brain. NeuroImage, 137, 21-33.

King, J. R., \& Dehaene, S. (2014). Characterizing the dynamics of mental representations: The temporal generalization method. Trends in Cognitive Sciences, 18, 203-210.

Korn, E. L., Troendle, J. F., Mcshane, L. M., \& Simon, R. (2004). Controlling the number of false discoveries: application to highdimensional genomic data. Journal of Statistical Planning and Inferences, 124, 379-398.

Kriegeskorte, N., Goebel, R., \& Bandettini, P. (2006). Information-based functional brain mapping. Proceedings of the National Academy of Sciences of the United States of America, 103, 3863-3868.

Kriegeskorte, N., Mur, M., \& Bandettini, P. (2008). Representational similarity analysis - connecting the branches of systems neuroscience. Frontiers in Systems Neuroscience, 2, 4.

Kriegeskorte, N., Simmons, W. K., Bellgowan, P. S., \& Baker, C. I. (2009). Circular analysis in systems neuroscience: the danger of double dipping. Nature Neuroscience, 12, 535-540.

Lopez-Calderon, J., \& Luck, S. (2014). ERPLAB: an open-source toolbox for the analysis of event-related potentials. Frontiers in Human Neuroscience, 8, 213. https://doi.org/10.3389/fnhum.2014.00213.

Luck, S. J. (2005). An introduction to the event-related potential technique. Cambridge: MIT.

Maris, E., \& Oostenveld, R. (2007). Nonparametric statistical testing of EEG- and MEG-data. Journal of Neuroscience Methods, 164, 177 190.

Meyers, E. M., \& Kreiman, G. (2011). Tutorial on pattern classification in cell recordings. In N. Kriegeskorte \& G. Kreiman (Eds.), Understanding visual population codes. Boston: MIT Press.

Meyers, E. M., Freedman, D. J., Kreiman, G., Miller, E. K., \& Poggio, T. (2008). Dynamic population coding of category information in inferior temporal and prefrontal cortex. Journal of Neurophysiology, 100, 1407-1419.

Müller, K. R., Krauledat, M., Dornhege, G., Curio, G., \& Blankertz, B. (2004). Machine learning techniques for brain-computer interfaces. Biomedical Technology, 49, 11-24.

Müller, K. R., Tangermann, M., Dornhege, D., Krauledat, M., Curio, G., \& Blankertz, B. (2008). Machine learning for real-time single-trial EEG-analysis: From brain-computer interfacing to mental state monitoring. Journal of Neuroscience Methods, 167, 82-90.

Mur, M., Bandettini, P. A., \& Kriegeskorte, N. (2009). Revealing representational content with pattern- information $\mathrm{fMRI}$ - an introductory guide. Social Cognitive and Affective Neuroscience, 4, 101-109.

Nili, H., Wingfield, C., Walther, A., Su, L., Marslen-Wilson, W., \& Kriegeskorte, N. (2014). A toolbox for representational similarity analysis. PLoS Computational Biology, 10, e1003553.
Norman, K. A., Polyn, S. M., Detre, G. J., \& Haxby, J. V. (2006). Beyond mind-reading: multi-voxel pattern analysis of fMRI data. Trends in Cognitive Sciences, 10, 424-430.

Oostenveld, R., Fries, P., Maris, E., and Schoffelen, J.-M. (2011). FieldTrip: Open source software for advanced analysis of MEG, EEG, and invasive electrophysiological data. Computational Intelligence and Neuroscience, (2011). https://doi.org/10.1155/ 2011/156869.

Oosterhof, N. N., Connolly, A. C., \& Haxby, J. V. (2016). CoSMoMVPA: Multi-modal multivariate pattern analysis of neuroimaging data in Matlab/GNU Octave. Frontiers in Neuroinformatics, 10, 27. https:// doi.org/10.3389/fninf.2016.00027.

Parra, L. C., Alvino, C., Tang, A., Pearlmutter, B., Yeung, N., Osman, A., \& Sajda, P. (2002). Linear spatial integration for single-trial detection in encephalography. NeuroImage, 17, 223-230.

Parra, L. C., Spence, C. D., Gerson, A. D., \& Sajda, P. (2005). Recipes for the linear analysis of EEG. NeuroImage, 28, 326-341.

Philiastides, M. G., \& Sajda, P. (2006). Temporal characterization of the neural correlates of perceptual decision making in the human brain. Cerebral Cortex, 16, 509-518.

Philiastides, M. G., Ratcliff, R., \& Sajda, P. (2006). Neural representation of task difficulty and decision making during perceptual categorization: a timing diagram. The Journal of Neuroscience, 26, 8965-8975.

Quek, G.L., and Rossion, B. (2017). Category-selective human brain processes elicited in fast periodic visual stimulation streams are immune to temporal predictability. Neuropsychologia, 104, 182-200. https://doi.org/10.1101/117135.

Ratcliff, R., Philiastides, M. G., \& Sajda, P. (2009). Quality of evidence for perceptual decision making is indexed by trial-to-trial variability of the EEG. Proceedings of the National Academy of Sciences of the United States of America, 106, 6539-6544.

Sajda, P., Philiastides, M. G., \& Parra, L. C. (2009). Single-trial analysis of neuroimaging data: inferring neural networks underlying perceptual decision-making in the human brain. IEEE Reviews in Biomedical Engineering, 2, 97-109.

Schrouff, J., Rosa, M. J., Rondina, J. M., Marquand, A. F., Chu, C., Ashburner, J., et al. (2013). PRoNTo: Pattern recognition for neuroimaging toolbox. Neuroinformatics, 11, 319-337.

Stelzer, J., Chen, Y., \& Turner, R. (2013). Statistical inference and multiple testing correction in classification-based multi-voxel pattern analysis (MVPA): Random permutations and cluster size control. NeuroImage, 65, 69-82.

Stokes, M. G., Wolff, M. J., \& Spaak, E. (2015). Decoding rich spatial information with high temporal resolution. Trends in Cognitive Sciences, 19, 636-638.

Tong, F., \& Pratte, M. S. (2012). Decoding patterns of human brain activity. Annual Review of Psychology, 63, 483-509.

Turner, W. F., Johnston, P., de Boer, K., Morawetz, C., \& Bode, S. (2017). Multivariate pattern analysis of event-related potentials predicts the subjective relevance of everyday objects. Consciousness and Cognition.

Tzovara, A., Chavarriaga, R., and De Lucia, M. (2015). Quantifying the time for accurate EEG decoding of single value-based decisions. Journal of Neuroscience Methods, 250, 114-125.

Van Vugt, M. K., Simen, P., Nystrom, L. E., Holmes, P., \& Cohen, J. D. (2012). EEG oscillations reveal neural correlates of evidence accumulation. Frontiers in Neuroscience, 6, 106. https://doi.org/10. 3389/fnins.2012.00106.

Wilcox, R. (2012). Introduction to Robust Estimation and Hypothesis Testing. Waltham MA: Academic Press.

Woolgar, A., Golland, P., \& Bode, S. (2014). Coping with confounds in multi-voxel analysis: what should we do about reaction time differences? A comment on Todd, Nystrom \& Cohen 2013. NeuroImage, 98, 73-80.

Woolgar, A., Jackson, J., \& Duncan, J. (2016). Coding of visual, auditory, rule, and response information in the brain: 10 years of multivoxel 
pattern analysis. Journal of Cognitive Neuroscience, 28, 14331454.

Wyart, V., de Gardelle, V., Scholl, J., and Summerfiled, C. (2012). Rhythmic fluctuations in evidence accumulation during decision making in the human brain. Neuron, 76, 847-858.

Wyart, V., Myers, N. E., \& Summerfield, C. (2015). Neural mechanisms of human perceptual choice under focussed and divided attention. The Journal of Neuroscience, 35, 3485-3498.
Yarkoni, T., and Westfall, J. (2017). Choosing prediction over explanation in psychology: Lessons from machine learning. Perspectives in Psychological Science, 12(6), 1100-1122. https://doi.org/10.1177/ 1745691617693393

Yuen, K. K. (1974). The two-sample trimmed t for unequal population variances. Biometrika, 61, 165-170. 